Published in final edited form as:

Int J Cancer. 2016 January 1; 138(1): 187-194. doi:10.1002/ijc.29686.

\title{
Therapeutic administration of IL-15 superagonist complex ALT-803 leads to long-term survival and durable antitumor immune response in a murine glioblastoma model
}

\author{
Dimitrios Mathios ${ }^{1}{ }^{,}$, Chul-Kee Park ${ }^{1,2,{ }^{*}}$, Warren D. Marcus ${ }^{3}$, Sarah Alter $^{3}$, Peter R. Rhode ${ }^{3}$, \\ Emily K. Jeng ${ }^{3}$, Hing C. Wong ${ }^{3}$, Drew M. Pardoll ${ }^{4}$, and Michael Lim ${ }^{1}$ \\ ${ }^{1}$ Johns Hopkins University School of Medicine, Department of Neurosurgery, Baltimore, MD \\ ${ }^{2}$ Seoul National University College of Medicine, Department of Neurosurgery, Seoul, Republic of \\ Korea \\ ${ }^{3}$ Altor BioScience Corporation, Miramar, FL \\ ${ }^{4}$ Johns Hopkins University School of Medicine, Department of Oncology and Sidney Kimmel \\ Comprehensive Cancer Center, Baltimore, MD
}

\begin{abstract}
Glioblastoma is the most aggressive primary central nervous system malignancy with a poor prognosis in patients. Despite the need for better treatments against glioblastoma, very little progress has been made in discovering new therapies that exhibit superior survival benefit than the standard of care. Immunotherapy has been shown to be a promising treatment modality that could help improve clinical outcomes of glioblastoma patients by assisting the immune system to overcome the immunosuppressive tumor environment. Interleukin-15 (IL-15), a cytokine shown to activate several effector components of the immune system, may serve as an excellent immunotherapeutic candidate for the treatment of glioblastoma. Thus, we evaluated the efficacy of an IL-15 superagonist complex (IL-15N72D:IL-15RaSu-Fc; also known as ALT-803) in a murine GL261-luc glioblastoma model. We show that ALT-803, as a single treatment as well as in combination with anti-PD-1 antibody or stereo-tactic radiosurgery, exhibits a robust antitumor immune response resulting in a prolonged survival including complete remission in tumor bearing mice. In addition, ALT-803 treatment results in long-term immune memory against glioblastoma tumor rechallenge. Flow cytometric analysis of tumor infiltrating immune cells shows that ALT-803 leads to increased percentage of CD8+-cell infiltration, but not the NK cells, and IFN- $\gamma$ production into the tumor microenvironment. Cell depletion studies, in accordance with the flow cytometric results, show that the ALT-803 therapeutic effect is dependent on CD4+ and CD8+
\end{abstract}

Correspondence to: M. Lim, MD, Johns Hopkins Hospital, 600 N. Wolfe Street, Neurosurgery - Phipps 123, Baltimore, Maryland 21287, USA, Tel.: +1-410-614-1627, Fax: +1-410-502-4954, mlim3@jhmi.edu.

ND.M. and C.K.P. contributed equally to this work

Conflict of Interest: Hing C. Wong, Peter R. Rhode, Warren D. Marcus, Sarah Alter, and Emily K. Jeng are the employees and stockholders of Altor Bioscience Corporation.

Drew M. Pardoll: Research Funding: Bristol Myer Squibb

Michael Lim: Research Funding: Bristol Myer Squibb, Celldex Therapeutics, Immunocellular Therapeutics Ltd, Altor BioScience Corporation, Agenus, Merck, and Accuray. 
cells. These results provide a rationale for evaluating the therapeutic activity of ALT-803 against glioblastoma in the clinical setting.

\section{Keywords}

IL-15 superagonist; ALT-803; anti-PD-1; glioblastoma

Glioblastoma is the most frequent malignant brain tumor with an incidence of 3.2 per 100,000 person-years in the USA. ${ }^{1}$ Current standard management of glioblastoma (Stupp protocol) has shown only modest results providing treated patients with a median survival of 14.6 months and median time to tumor recurrence of 6.9 months. $^{2}$ Preclinical studies ${ }^{3,4}$ as well as human studies ${ }^{5,6}$ have shown that glioblastoma causes systemic suppression of patients' anti-tumor immune response. Furthermore, increased immunosuppression as measured by CD4+ and CD8+ T cell counts in patients with glioblastoma are associated with early death from tumor progression. ${ }^{7,8}$ These studies indicate that the immune system can play an important role in patient outcome, and support the use of cancer immunotherapy strategies that augment anti-tumor immune responses and/or relieve immunosuppression for more effective and durable control of glioblastoma.

Cancer cells are able to turn on various immune inhibitory pathways which are regulated by molecules and ligands such as PD- 1 and CTLA-4. ${ }^{9}$ Antibodies that block these checkpoint molecules have been shown to enhance anti-tumor immunity. Similar to ALT-803, antiPD-1 Ab targets a variety of innate and adaptive immune effector cells and increases their survival and proliferation. ${ }^{9}$ Anti-PD-1 Abs have already been used successfully in clinical trials for advanced metastatic tumors and were recently FDA approved for treatment of metastatic melanoma and squamous non-small-cell lung cancer. ${ }^{10}$

Radiation remains the main treatment approach for glioblastoma, and stereotactic radiosurgery has been implemented with great success in glioblastoma mouse models in our laboratory and in others as an immunostimulatory strategy. ${ }^{11-13}$ Using these models, it has also been demonstrated in a number of studies that the combination of SRS with immune checkpoint blockers has significant synergistic survival benefit in mice harboring glioblastoma tumors by increasing antigen presentation and promoting a proinflammatory tumor microenvironment. ${ }^{11-14}$ Therefore, it may be beneficial to combine SRS with other immunomodulators, such as IL-15.

Interleukin-15 (IL-15) is a four-helix common $\gamma$-chain cytokine that plays a crucial role in the development, maturation, and activation of NK cells and CD8+ memory T cells, thus has a potential use in cancer immunotherapy. ${ }^{15} \mathrm{IL}-15$ interacts with the IL-15 receptor a (IL-15Ra) on the surface of monocytes/macrophages and dendritic cells and is transpresented to the IL-2/IL-15 receptor $\beta$-common $\gamma$ chain (IL-15R $\beta \gamma_{c}$ ) complexes displayed on T cells and NK cells. ${ }^{15}$ Similar to IL-2, IL-15 activates and expands NK and CD8+ cells while increasing their cytolytic activity. Unlike IL-2 though, IL-15 does not expand the T regulatory population involved in suppressing immune responses and also does not induce activation-induced cell death of the CD8+ cells. ${ }^{15} \mathrm{IL}-15$ also sensitizes the T cell receptor signaling and increases the infiltration of CD8+ T cells. ${ }^{16}$ More importantly, IL-15 treatment 
does not cause significant capillary leak syndrome (hypotension, edema, and low levels of protein in the blood), ${ }^{17}$ a major side effect associated with IL-2 therapy. ${ }^{18}$ Thus, there is a strong rationale for the use of IL-15 over IL-2 as a superior immunostimulatory cytokine for cancer treatment and especially for gliomas as shown recently. ${ }^{19}$ However, the therapeutic efficacy of IL-15 in cancer had not been tested until recently, partially due to difficulties in the production of IL-15 and its short half-life and low biological activity in vivo. ${ }^{20}$

To overcome these limitations, an IL-15 superagonist complex (referred to as ALT-803) has been designed consisting of a novel human IL-15 superagonist variant (IL-15N72D) in a complex with a human IL-15Ra sushi domain-Fc fusion protein. ${ }^{20,21}$ It has previously been demonstrated that the IL-15N72D mutation increases IL-2R $\beta$ binding and IL-15 biological activity by approximately fivefold. ${ }^{22}$ Moreover, association of IL-15N72D to the soluble sushi domain of the IL-15Ra further increases the activity of ALT-803 based on enhanced binding to the IL-15R $\beta \gamma_{c}$ complex. ${ }^{22}$ The ALT-803 superagonist complex (IL-15N72D:IL-15RaSu/Fc) exhibits superior immunostimulatory activity, a longer serum half-life and retention in lymphoid organs, and significantly more potent efficacy against solid and hematologic tumors compared to IL-15 in mouse models. ${ }^{22,23}$ Currently, ALT-803 is being evaluated in multiple clinical trials against hematological and solid malignancies based on encouraging result of in vivo studies. ${ }^{22,23}$

In this article, we further developed these studies by evaluating the antitumor activity and mechanism of action of ALT-803 alone and in combination with checkpoint blockade or stereotactic radiosurgery in a syngeneic orthotopic murine glioblastoma model with the goal of establishing the optimal immunotherapeutic regimen for human clinical study against glioblastoma.

\section{Materials and Methods}

\section{Mice and tumor cell lines}

C57BL/6J female mice (6-8 weeks old) were obtained from Jackson Laboratories (Bar Harbor, ME, USA). All animal studies were conducted according to the NIH animal care guidelines under an approved Institutional Animal Care and Use Committee protocol from Johns Hopkins University.

GL261-luc murine glioblastoma cell line was purchased from Perkin Elmer (Waltham, MA, USA). The cells were cultured in Dulbecco's Modified Eagle Medium (Life Technologies, Frederick, MD, USA) with the addition of $10 \%$ FBS (Thermo Scientific, Waltham, MA, USA), $1 \%$ P/S (Thermo Scientific, Waltham, MA, USA), and $100 \mu \mathrm{g} / \mathrm{ml}$ of G418 (Invivogen, San Diego, CA, USA). The cells were allowed to grow in a humidified incubator at $37^{\circ} \mathrm{C}$ with $5 \% \mathrm{CO}_{2}$.

\section{Tumor model}

Mice were deeply anesthetized with ketamine/xalizine $(100 \mathrm{mg} / \mathrm{kg}$ ketamine $/ 10 \mathrm{mg} / \mathrm{kg}$ xylazine), their skull skin was prepped with betadine, and an incision was made in the midline. After identifying the sagittal and lambdoid sutures on the left side, a hole was drilled at the following coordinates: $1 \mathrm{~mm}$ anterior and $1 \mathrm{~mm}$ lateral from bregma. GL261-luc tumor 
cells $(130,000$ in $1 \mu$ of PBS) were injected with a Hamilton syringe loaded on a stereotactic machine in the left striatum of the mouse by guiding the needle $3 \mathrm{~mm}$ deep from the surface of the dura. The skin incision was closed and mice were monitored until they completely recovered from anesthesia.

For the rechallenge experiments, the above-mentioned protocol was used but the right side (contralateral to initial implantation) was drilled. 300,000 GL261-luc cells were implanted. Mice were assessed with in vivo bioluminescent imaging every 3 days for the first week and weekly onward for tumor progression and followed for signs of neurologic deterioration daily. Mice were injected i.p. with $300 \mu$ of luciferin (Perkin Elmer, Waltham, MA, USA). The luciferin was allowed to circulate for $5 \mathrm{~min}$ before the mice were anesthetized with a mix of $\mathrm{O}_{2}$ and isoflurane (2.5\%). Mice that showed an increase in tumor burden based on imaging during the first week after tumor implantation were included in the study. Mice were stratified on Day 7 into our treatment arms based on their bioluminescent signal.

ALT-803 (Altor Bioscience Corporation, Miramar, FL, USA) was given via intraperitoneal (i.p.) injections at a dose of $0.25 \mathrm{mg} / \mathrm{kg}$. A variety of therapeutic schedules were used to identify whether the timing of administration or the frequency of administration would impact the final outcome.

\section{In vivo depletion studies}

For in vivo depletion of NK1.1, CD4+, and CD8+ cells, mice were injected i.p. with $200 \mu \mathrm{g} /$ dose anti-NK1.1 (clone PK136, ATCC), $200 \mu \mathrm{g} / \mathrm{dose}$ anti-CD4 (clone GK1.5), and $500 \mu \mathrm{g} /$ dose anti-CD8 (clone 53-6.72 ATCC) antibodies (Abs), respectively. Control mice received PBS $(0.2 \mathrm{ml})$. Antibodies were given to tumor-bearing mice $48 \mathrm{hr}$ and $24 \mathrm{hr}$ before the first dose of ALT-803, and reinjected every 7 days for 3 weeks. The efficiency of CD4+, CD8+, and NK1.1 cell depletion was assessed by flow cytometry of peripheral blood monocyteslymphocytes.

\section{Flow cytometry}

The immunologic profile of tumor infiltrating lymphocytes (TILs) was assessed on day 18 after GL261-luc tumor implantation. Brains of tumor bearing mice from both the ALT-803treated and untreated mice were harvested after perfusing the mice with PBS (via intracardiac injection) to remove any possible artifact of circulating lymphocytes in the brain. Tumor infiltrating lymphocytes were separated from tumor cells and myelin by using a three-level Percoll Gradient (30\%, 37\%, and 60\%). Tumor infiltrating lymphocytes were isolated from the interface of the 37-60\% layers. Following a wash with PBS, cells were stimulated with PMA/ ionomyocin in the presence of Golgi stop and Monesin (eBioscience, San Diego, CA, USA) for $4 \mathrm{hr}$. Cells were washed with PBS and then stained with Aqua Live/Dead stain (Life Technologies, Frederick, MD, USA) and fluoro-conjugated Abs specific to cell surface markers CD3 (clone 17A2, FITC), CD4 (clone GK1.5, APCH7), CD8 (clone 53-6.7, PerCPCy5.5), and NK1.1 (clone PK136, PECF594) (eBio-science, BD Biosciences, San Jose, CA, USA). After fixation-permeabilization (Fixation/ Permeabilization buffer, eBio-science), cells were stained with Abs specific to IFN $\gamma$ (clone 
XMG 1.2, APC) and FoxP3 (clone MF23, PE) (eBioscience) or with the isotype control Abs (rat IgG1k-APC for IFN $\gamma$ and rat IgG2b-PE for FoxP3).

\section{Anti-PD-1 antibody (ab) treatment}

Hamster anti-murine PD-1 Ab-producing hybridoma (G4) was used to produce the antibody as previously described. ${ }^{24}$ Anti-PD1 Ab treatment was given at $200 \mu \mathrm{g} / \mathrm{dose}$ intraperitoneally. Hamster immunoglobulin isotype (Rockland Immuno-chemicals Inc., Gilbertsville, PA, USA) Ab was administered as a control.

\section{Stereotactic radiation}

The Small Animal Radiation Research platform (SARRP, Xstrahl, UK) was used at the Johns Hopkins University Radiation Core facility for the radiation experiments as previously described. ${ }^{11}$ Briefly, a loaded CT imaging system was used initially to scan the head of the mouse to identify the hole created at the time of implantation and the radiation beam isocenter was set $3 \mathrm{~mm}$ below the inner plate of the skull starting from the center of the burr hole. A single $10 \mathrm{~Gy}$ radiation dose was delivered at the tumor for the radiation treatment groups.

\section{Statistical analysis}

Kaplan-Meier survival analysis was performed to assess survival differences among the treatment groups and $p$ values were calculated with the log-rank test. A two-tailed Student's $t$ test, was used to calculate $p$ values when assessing continuous variables. Statistical significance was accepted at a level of $p \leq 0.05$.

\section{Results}

\section{ALT-803 shows durable anti-tumor effect in a murine glioblastoma model}

Previous studies have demonstrated potent immune cell-mediated antitumor activities of ALT-803 treatment in syngeneic mouse models of solid and hematologic tumors. ${ }^{22,23}$

To evaluate whether ALT-803 is also efficacious against aggressive brain tumors, we used mice that had been intracranially implanted with the glioblastoma cell line, GL261-luc. As shown in Figure 1, treatment with ALT-803 was initiated 7 or 10 days postimplantation when tumors were well established. The dose frequency of ALT- 803 was also varied ( 2 or 3 doses) to determine its effect on therapeutic activity. In PBS-treated control mice, the intracranial GL261-luc tumor burden progressively increased resulting in animal deaths approximately 3 weeks post-tumor instillation. Treatment of glioblastoma-bearing mice with ALT-803 beginning at either day 7 or day 10 after tumor implantation resulted in inhibition of tumor growth. Mice treated with 2 or 3 doses of ALT-803 exhibited statistically significant increases in survival compared to the control mice (median survival: PBS, 25.5 days; 2-dose ALT-803, 31 days, $P=0.03$; three-dose ALT-803, 35 days, $P=0.02$ vs PBS, $P$ $=0.5$ vs 2 dose ALT-803). The time of treatment initiation (day $7 v s$ day 10) did not seem to affect treatment outcome ( $P=0.8)$ (Fig. 1). Therefore, ALT-803 treatment of glioblastomatumor-bearing mice reduced tumor burden and significantly prolonged survival regardless of 
time of treatment initiation or the number of doses administered. None of the mice exhibited signs of toxicity such as weigh loss or hair loss.

\section{ALT-803 in combination with anti-PD-1 Ab showed additive anti-tumor effect}

Anti-PD-1 Abs have already been used successfully in clinical trials for advanced metastatic tumors and were recently FDA approved for treatment of metastatic melanoma and squamous non-small-cell lung cancer. ${ }^{10}$ The combination of ALT-803 and anti-PD-1 Ab was evaluated to determine if there was increased efficacy of these molecules in the GL261luc glioblastoma mouse model. Treatment with multiple doses of ALT-803 (3 or 4 doses) or anti-PD-1 Ab (3 doses) as monotherapy exhibited similar increases in antitumor activity and prolonged animal survival when compared to PBS-treated controls (median survival: PBS, 16 days; three-dose ALT-803, 19 days, $P=0.02$ vs PBS; four-dose ALT-803, 23 days, $P$ $=0.0004$ vs PBS, three-dose anti-PD- 1 Ab, 25.5 days, $P=0.0004$ vs PBS). The combination of ALT-803 and anti-PD1 Ab treatment further extended median survival times of tumorbearing mice (median survival: three-dose ALT-803 +anti-PD-1 Ab, 26 days, $P=0.001 v s$ PBS; four-dose ALT-803 +anti-PD-1 Ab, 28.5 days, $P=0.002$ vs PBS). It was also found that anti-PD-1 Ab in combination with 4 doses of ALT-803 increased the percentage of long-term survivors ( $>60$ days postimplantation) to $40 \%$ from the $20 \%$ rate observed in mice treated with anti-PD-1 Ab and ALT-803 monotherapy (Fig. 2). These results suggest that combining the immunostimulatory activity of ALT-803 with the checkpoint blocker, antiPD-1 Ab, has a beneficial additive effect in prolonging survival of glioblastoma tumor bearing mice.

\section{ALT-803 generate anti-tumor immune memory}

Mice that exhibited no sign of tumor by IVIS imaging by day 60 were rechallenged with GL261 luc cells in the contralateral from the initial implantation hemisphere. All the 12 survived mice after the treatment of ALT-803 or anti-PD-1 monotherapy, and ALT-803/ anti-PD-1 combination therapy mounted an immune memory response against the tumor cells preventing tumor formation upon rechallenge (Fig. 2).

\section{ALT-803 in combination with stereotactic radiosurgery (SRS)}

As shown in Figure 3, ALT-803 alone or in combination with SRS exhibited an increased survival trend compared to the control mice (20\% of ALT-803 and 30\% of ALT-803/SRS combination). However, SRS did not have a synergistic or additive survival benefit with ALT-803 compared to ALT-803 monotherapy (Fig. 3).

\section{Anti-tumor effect of ALT-803 is mediated by CD4+ and CD8+ T cells}

Previous studies of ALT-803 in different tumor models have shown that different subsets of immune cells are important modulators of anti-tumor immunity. For example, in the syngeneic murine model of multiple myeloma, ALT-803 treatment was CD8+ T-celldependent, ${ }^{22}$ whereas in the bladder tumor model, ALT-803 treatment led to NK cell expansion. ${ }^{23}$ In this study, the immune cell populations responsible for the therapeutic benefit conferred by ALT-803 treatment were determined through cell depletion studies. Antibodies against CD4, CD8, and NK were used to deplete specific immune cell subsets. 
Both CD4 and CD8 depletion in GL261 tumor-bearing mice abrogated the ALT-803 treatment-related survival benefit, whereas NK depletion did not affect the survival of ALT-803-treated mice (Fig. 4). This implies that NK cells, despite infiltrating the tumor (as shown below), do not play a crucial role in mediating the ALT-803 therapeutic benefit. These results confirm that the immune activating ability of ALT- 803 is broad and able to induce different arms of the immune system in the appropriate tumor model settings.

\section{Flow cytometric analysis of tumor infiltrating lymphocytes}

The phenotypic identity of immune cell subsets within the tumors of ALT-803-treated mice was determined by flow cytometry. The immune cell infiltrates in mice treated with ALT-803 had a higher enrichment in CD3+ cells than NK cells when compared to the nontreated control mice as shown by the CD3/NK1.1 ratio ( 8 vs $2, p=0.1$ ) (Fig. $5 \mathrm{~A}$ ). A trend toward an increase in the percentage of tumor-infiltrating CD8+ lymphocytes was also observed when comparing ALT- 803 treated and nontreated mice. Among the infiltrating T cells extracted from the ALT-803-treated mice, the percentage of CD8+ cells producing IFN $\gamma$ upon stimulation was higher compared to the nontreated group $(1.8 \%$ vs $0.8 \%, p$ $=0.05)$. Conversely, the percentage of $\mathrm{T}_{\text {reg }} \mathrm{s}(\mathrm{CD} 4+\mathrm{FoxP} 3+)$ was decreased in the ALT-803treated group compared to the nontreated group (Fig. 5B). When looking at the $\mathrm{T}_{\text {eff }} / \mathrm{T}_{\text {reg }}$ ratio, the ALT-803-treated mice had a higher ratio compared to nontreated mice (6 vs $0.1, p$ $=0.2)($ Fig. $5 \mathrm{~B})$. These results are consistent with the role of CD8+ cells in survival benefit observed following ALT-803 treatment of tumor-bearing mice.

\section{Discussion}

In this study, administration of ALT-803 in Gl261 tumor-bearing mice was found to inhibit tumor growth and prolong survival. Additionally, tumor eradication was observed in approximately $20 \%$ of the ALT- 803 treated mice resulting in long-term survivors. In this model, three doses of ALT- 803 produced the best objective responses but dosing with as low as 2 doses was enough to produce statistically significant survival benefit compared to the nontreated mice $(p=0.03$ ). Tumor eradication was rapid, with complete responders losing their BLI tumor signal 4 days after initiation of ALT-803 treatment.

ALT803's size does not allow for diffusion of the drug through the blood-brain barrier (BBB). However, our approach circumvents the obstacle of the BBB, as ALT803 is not necessary to work in situ (within the tumor microenvironment) to activate the immune system. Instead it is activating the peripheral immune system (lymph nodes, spleen, bone marrow) by allowing for proliferation and longer life span of the innate and adaptive immune response that can then infiltrate the tumor microenvironment. These activated immune cells can readily pass the BBB and act on their target.

The ALT-803 therapeutic effect seemed to be mediated through both the CD4+ and CD8+ T cells, but not the NK cells. However, we acknowledge that NK cells could potentially play a role in other systems. This result is in agreement with previously published data where ALT-803 therapeutic efficacy against multiple myeloma is mediated through CD8+ T cells, but not NK cells. ${ }^{21,22}$ These data were also confirmed via flow cytometry studies, which demonstrated an increase in the ratio of CD3:NK1.1 cells, as well as the CD8+ IFN $\gamma$ - 
producing $\mathrm{T}$ cells. It is different though from other tumor models, such as bladder cancer, where it has been shown that NK cells are the mediators of ALT-803 anti-tumor effect.

It has been consistently shown that the major player of immune-mediated antitumor response in glioblastoma is CD4 cells and secondarily CD8 cells. ${ }^{7,25-27}$ Despite their presence in brain tumor immune infiltrates, NK cells have consistently been shown not to play a central role in brain tumor establishment or progression and more importantly clinical trials that harness the NK power did not show much promise. ${ }^{18,28,29}$ ALT-803 utilizing multiple mechanisms of action seems to potentiate the efficacy of the "dominant" population specific for every tumor model. Multiple myeloma does not seem to be affected by NK activity, whereas bladder cancer seems to be greatly affected by it. The biology of each tumor, the antigen presentation machinery used in response to the tumor, and the mechanism of immune potentiation likely differ with the different tumor models and tumor types. Furthermore, it is possible that there is a lack of ligands on the 5T33 cells (in the multiple myeloma model) and GL261-luc cells (in the glioblastoma model) to bind the activating receptors of the NK cells or that these tumor cells express ligands on strongly interact with inhibitory receptors on the NK cell surface (such as a Ly49:MHC-I interaction) and disrupt the ability of NK cells to kill these specific tumor cells. These possibilities could be explored in future studies to better understand the activity of NK cells against glioblastoma tumors.

Interestingly, CD4+ T cell depletion of mice with GL261-luc tumors abrogates the ALT-803 treatment-related survival benefit, a finding that has not been reported previously in mice with multiple myeloma- or melanoma-bearing mice treated with IL-15/IL-15Ra complexes. ${ }^{21,22}$ This result implies that in the glioblastoma model, CD4+ T cell activity is required to mount a robust anti-tumor response. $\mathrm{CD} 4+\mathrm{T}$ cells can exert their effector functions by either direct cyto-toxicity against tumor cells or by helping the CD8+ T cells at the time of priming to acquire a memory cell phenotype allowing for long-term maintenance of the effector CD8+ cells. The fact that both CD8+ and CD4+ T cell depletion completely abrogates the survival benefit of ALT-803 indicates that the latter theory is more probable. In the absence of CD8+ T cells, the CD4+ T cells cannot mount an immune response strong enough to reject the tumor as seen in animals treated with ALT-803 alone. The mice that completely responded to ALT-803 treatment rejected tumor rechallenge of GL261-luc cells. This was an anticipated result since a murine model of multiple myeloma demonstrated a similar effect. ${ }^{22}$ It is likely that antigens that remained in the microenvironment after tumor killing were then captured and presented to naive memory $\mathrm{T}$ cells by the antigen presenting cells, priming them for future effector functions against rechallenged tumor.

ALT-803 had an additive effect in combination with anti-PD-1 mAb on survival of GL261luc tumor-bearing mice. This was anticipated since ALT-803 did not affect the levels of PD-1 expression on lymphocytes ${ }^{20}$ and anti-PD-1 Ab acts in an alternative pathway to increase the function of exhausted effector cells.

In contrast, ALT-803, in combination with SRS, did not confer any additional survival benefit to either monotherapy. Stereotactic radiosurgery has been shown to increase the infiltration of CD8+ cells in the tumor microenvironment and has been used successfully in 
combination with several immune checkpoint inhibitors, including anti-PD-1, anti-CTLA4, and anti-4-1BB. ${ }^{11-13}$ The lack of synergism between SRS and ALT-803 could be explained by the mechanism in which ALT-803 and SRS are stimulating an immune response. It has been hypothesized that SRS acts to induce an immune response by increasing tumor antigen release and presentation which leads to expansion and activation of antigen specific T-cell clones against the tumor. ${ }^{13}$ As explained previously, the rapid antitumor activity of ALT-803 is not believed to be mediated by a TCR-dependent immune response; instead, it directly activates innate-like CD8+ memory cell cytotoxicity against the tumor. Despite having a different mechanism of action on the immune system, the final responding cell population following ALT-803 and SRS treatment is the CD8+ cytotoxic T cells, which may make these two treatment modalities redundant.

In summary, ALT-803 significantly extended the survival of glioblastoma bearing mice. The immunotherapeutic effect was CD4+ and CD8+ cell mediated. These preclinical data provide evidence that support the testing of ALT-803 in glioblastoma patients in a clinical trial setting.

\section{References}

1. Ostrom QT, Gittleman H, Liao P, et al. CBTRUS statistical report: primary brain and central nervous system tumors diagnosed in the United States in 2007-2011. Neuro-Oncology. 2014; 16(Suppl 4):iv1-63. [PubMed: 25304271]

2. Stupp R, Mason WP, van den Bent MJ, et al. Radiotherapy plus concomitant and adjuvant temozolomide for glioblastoma. The New England Journal of Medicine. 2005; 352:987-96. [PubMed: 15758009]

3. Chae M, Peterson TE, Balgeman A, et al. Increasing glioma-associated monocytes leads to increased intratumoral and systemic myeloid-derived suppressor cells in a murine model. Neuro oncology. 2015; 17:978-91. [PubMed: 25537019]

4. Authier A, Farrand KJ, Broadley KW, et al. Enhanced immunosuppression by therapy-exposed glioblastoma multiforme tumor cells. International Journal of Cancer. 2015; 136:2566-78.

5. Bloch O, Crane CA, Kaur R, et al. Gliomas promote immunosuppression through induction of B7H1 expression in tumor-associated macrophages. Clinical Cancer Research: an Official Journal of the American Association for Cancer Research. 2013; 19:3165-75. [PubMed: 23613317]

6. Brooks WH, Netsky MG, Normansell DE, et al. Depressed cell-mediated immunity in patients with primary intracranial tumors. Characterization of a humoral immunosuppressive factor. The Journal of Experimental Medicine. 1972; 136:1631-47. [PubMed: 4345108]

7. Grossman SA, Ye X, Lesser G, et al. Immuno-suppression in patients with high-grade gliomas treated with radiation and temozolomide. Clinical Cancer Research: an Official Journal of the American Association for Cancer Research. 2011; 17:5473-80. [PubMed: 21737504]

8. Prins RM, Soto H, Konkankit V, et al. Gene expression profile correlates with T-cell infiltration and relative survival in glioblastoma patients vaccinated with dendritic cell immunotherapy. Clinical Cancer Research: an Official Journal of the American Association for Cancer Research. 2011; 17:1603-15. [PubMed: 21135147]

9. Pardoll DM. The blockade of immune checkpoints in cancer immunotherapy. Nature Reviews Cancer. 2012; 12:252-64. [PubMed: 22437870]

10. Pardoll DM. Immunology beats cancer: a blueprint for successful translation. Nature Immunology. 2012; 13:1129-32. [PubMed: 23160205]

11. Zeng J, See AP, Phallen J, et al. Anti-PD-1 blockade and stereotactic radiation produce long-term survival in mice with intracranial gliomas. International Journal of Radiation Oncology, Biology, Physics. 2013; 86:343-9. 
12. Belcaid Z, Phallen JA, Zeng J, et al. Focal radiation therapy combined with 4-1BB activation and CTLA-4 blockade yields long-term survival and a protective antigen-specific memory response in a murine glioma model. PloS One. 2014; 9:e101764. [PubMed: 25013914]

13. Sharabi AB, Nirschl CJ, Kochel CM, et al. Stereo-tactic radiation therapy augments antigenspecific PD-1 mediated anti-tumor immune responses via cross-presentation of tumor antigen. Cancer Immunol Res. 2015; 3:345-55. [PubMed: 25527358]

14. Nicholas S, Mathios D, Ruzevick J, et al. Current trends in glioblastoma multiforme treatment: radiation therapy and immune checkpoint inhibitors. Brain Tumor Research and Treatment. 2013; 1:2-8. [PubMed: 24904882]

15. Waldmann TA. The biology of interleukin-2 and interleukin-15: implications for cancer therapy and vaccine design. Nature Reviews Immunology. 2006; 6:595-601.

16. Liu K, Catalfamo M, Li Y, et al. IL-15 mimics T cell receptor crosslinking in the induction of cellular proliferation, gene expression, and cytotoxicity in CD8+ memory T cells. Proc Natl Acad Sci U S A. 2002; 99:6192-7. [PubMed: 11972069]

17. Waldmann TA, Lugli E, Roederer M, et al. Safety (toxicity), pharmacokinetics, immunogenicity, and impact on elements of the normal immune system of recombinant human IL-15 in rhesus macaques. Blood. 2011; 117:4787-95. [PubMed: 21385847]

18. Mier JW, Brandon EP, Libby P, et al. Activated endothelial cells resist lymphokine-activated killer cell-mediated injury. Possible role of induced cytokines in limiting capillary leak during IL-2 therapy. Journal of Immunology. 1989; 143:2407-14.

19. Garofalo S, D'Alessandro G, Chece G, et al. Enriched environment reduces glioma growth through immune and non-immune mechanisms in mice. Nat Commun. 2015; 6:6623. [PubMed: 25818172]

20. Wong HC, Jeng EK, Rhode PR. The IL-15-based superagonist ALT-803 promotes the antigenindependent conversion of memory CD8 T cells into innate-like effector cells with antitumor activity. Oncoimmunology. 2013; 2:e26442. [PubMed: 24404427]

21. Epardaud M, Elpek KG, Rubinstein MP, et al. Interleukin-15/interleukin-15R alpha complexes promote destruction of established tumors by reviving tumor-resident CD8+ T cells. Cancer Research. 2008; 68:2972-83. [PubMed: 18413767]

22. Xu W, Jones M, Liu B, et al. Efficacy and mechanism-of-action of a novel superagonist interleukin-15: interleukin-15 receptor alphaSu/Fc fusion complex in syngeneic murine models of multiple myeloma. Cancer Research. 2013; 73:3075-86. [PubMed: 23644531]

23. Gomes-Giacoia E, Miyake M, Goodison S, et al. Intravesical ALT-803 and BCG treatment reduces tumor burden in a carcinogen induced bladder cancer rat model; a role for cytokine production and NK cell expansion. PloS One. 2014; 9:e96705. [PubMed: 24896845]

24. Hirano F, Kaneko K, Tamura H, et al. Blockade of B7-H1 and PD-1 by monoclonal antibodies potentiates cancer therapeutic immunity. Cancer Research. 2005; 65:1089-96. [PubMed: 15705911]

25. Segal BM, Glass DD, Shevach EM. Cutting Edge: IL-10-producing CD4+ T cells mediate tumor rejection. Journal of Immunology. 2002; 168:1-4.

26. Vetter M, Hofer MJ, Roth E, et al. Intracerebral interleukin 12 induces glioma rejection in the brain predominantly by CD8+ T cells and independently of interferon-gamma. J Neuropathol Exp Neurol. 2009; 68:525-34. [PubMed: 19525900]

27. Smith KE, Fritzell S, Badn W, et al. Cure of established GL261 mouse gliomas after combined immunotherapy with GM-CSF and IFNgamma is mediated by both CD8+ and CD4+ T-cells. International Journal of Cancer Journal International Du Cancer. 2009; 124:630-7. [PubMed: 18972433]

28. Dillman RO, Duma CM, Ellis RA, et al. Intralesional lymphokine-activated killer cells as adjuvant therapy for primary glioblastoma. J Immunother. 2009; 32:914. [PubMed: 19816190]

29. Jacobs SK, Wilson DJ, Melin G, et al. Interleukin-2 and lymphokine activated killer (LAK) cells in the treatment of malignant glioma: clinical and experimental studies. Neurol Res. 1986; 8:81-7. [PubMed: 2875409] 


\section{What's new?}

A new synthetic cytokine could jump-start the immunotherapy route to treating glioblastoma. The cytokine IL-15 has shown promise at stimulating an immune response, but its short half-life in the bloodstream has stymied efforts to deploy it against the deadly brain tumor. This article reports results from tests of a synthetic IL-15 molecule against glioblastoma in mice. They show that the molecule spurs a strong immune defense against the tumors, and a long-term immune memory against recurrence. Clinical testing in humans could be on the horizon. 

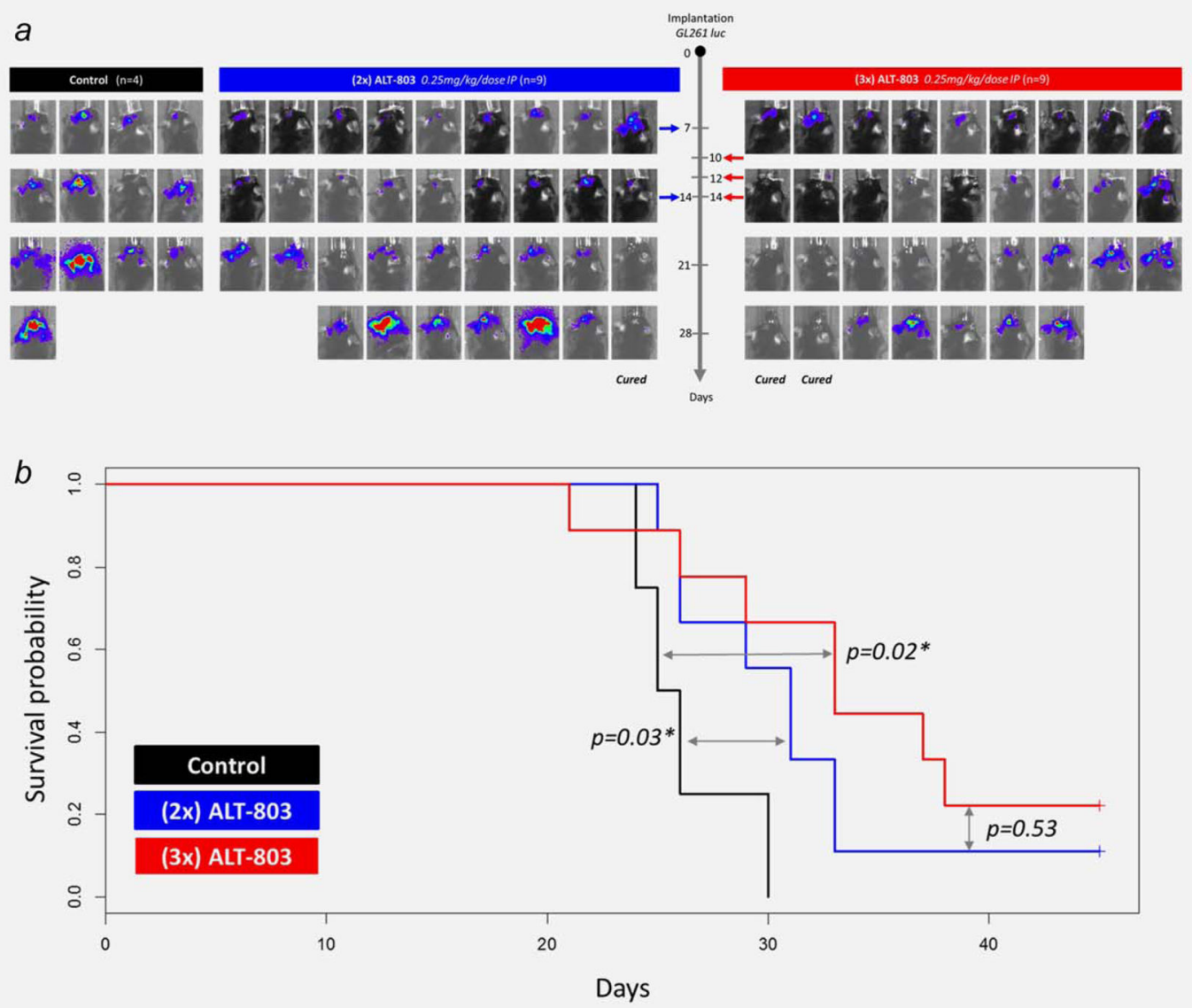

Figure 1.

Experimental schedule and survival outcome of ALT-803 administration. (A) Two different therapeutic schedules were tested: 2 doses (day 7 and 14) and 3 doses (day 10, 12, and 14) of ALT-803. The progression of tumor burden was assessed via IVIS imaging. In both ALT-803 treatment groups, inhibition of tumor growth was observed compared to controls with $10-20 \%$ of the ALT-803-treated animals exhibiting complete tumor eradication. (B) Kaplan-Meier survival curves of ALT-803 treated mice bearing glioblastoma tumors. ALT-803 monotherapy $(2 \times$ or $3 \times$ ) exhibited a statistically significant increase in the survival of mice compared to the PBS control group. Three doses of ALT-803 provided the longest median overall survival and resulted in $22 \%$ of the mice as long-term survivors. [Color figure can be viewed in the online issue, which is available at wileyonlinelibrary.com.] 

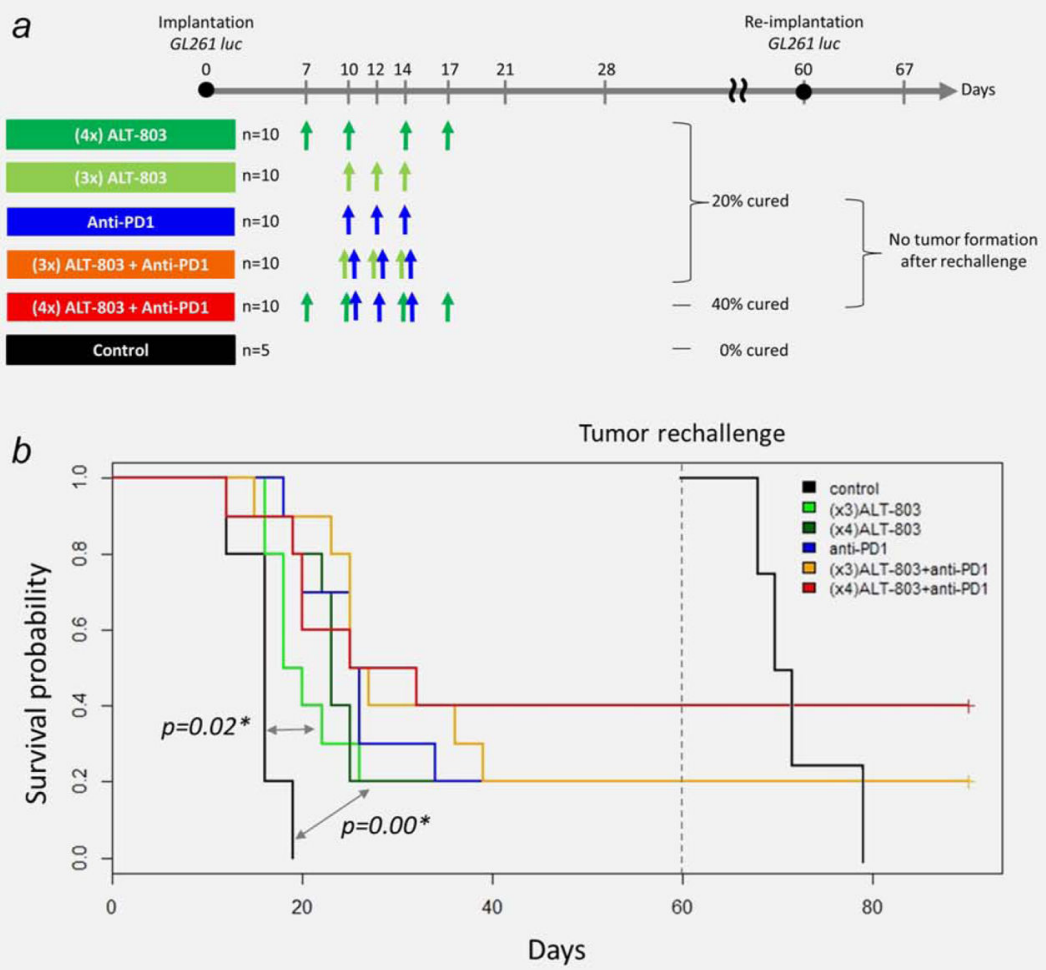

Figure 2.

(A) Administration of ALT-803 (either 3 doses or 4 doses) or anti-PD-1 antibody (3 doses) produced $20 \%$ of long-term survivor mice, and their combination increased to $40 \%$ longterm survival. Anti-tumor immune memory was maintained upon tumor rechallenge. (B) Kaplan-Meier survival curves. ALT-803 (4x)+anti-PD-1 Ab therapy provided the longest median survival time and produced the greatest percentage of long-term survivors (40\%) compared to monotherapies (ALT-803-20\%, anti-PD-1-20\%). [Color figure can be viewed in the online issue, which is available at wileyonlinelibrary.com.] 


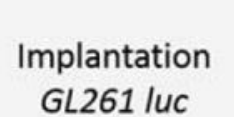

\section{(3x) ALT-803}

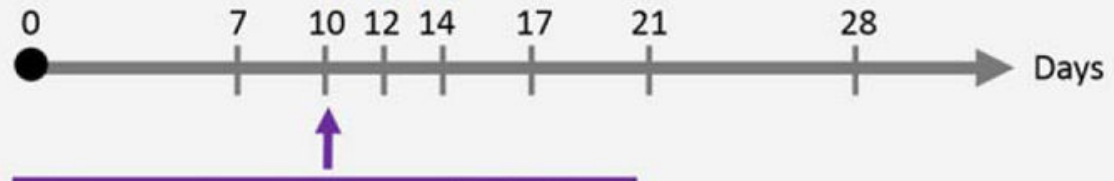

Stereotactic radiosurgery (SRS) 10Gy

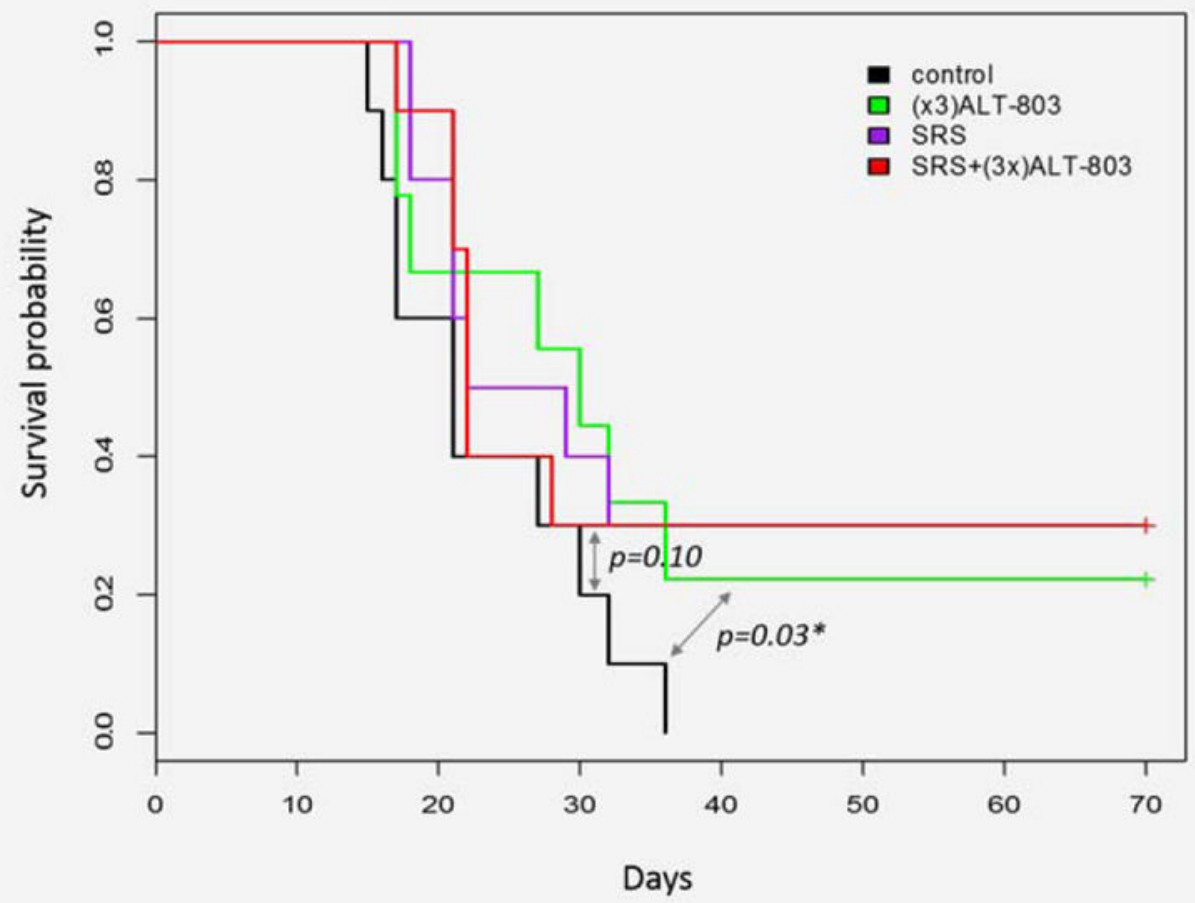

Figure 3.

ALT-803 has similar survival benefit with stereotactic radiosurgery (SRS) alone but the combination of the treatments does not exhibit synergism. [Color figure can be viewed in the online issue, which is available at wileyonlinelibrary.com.] 


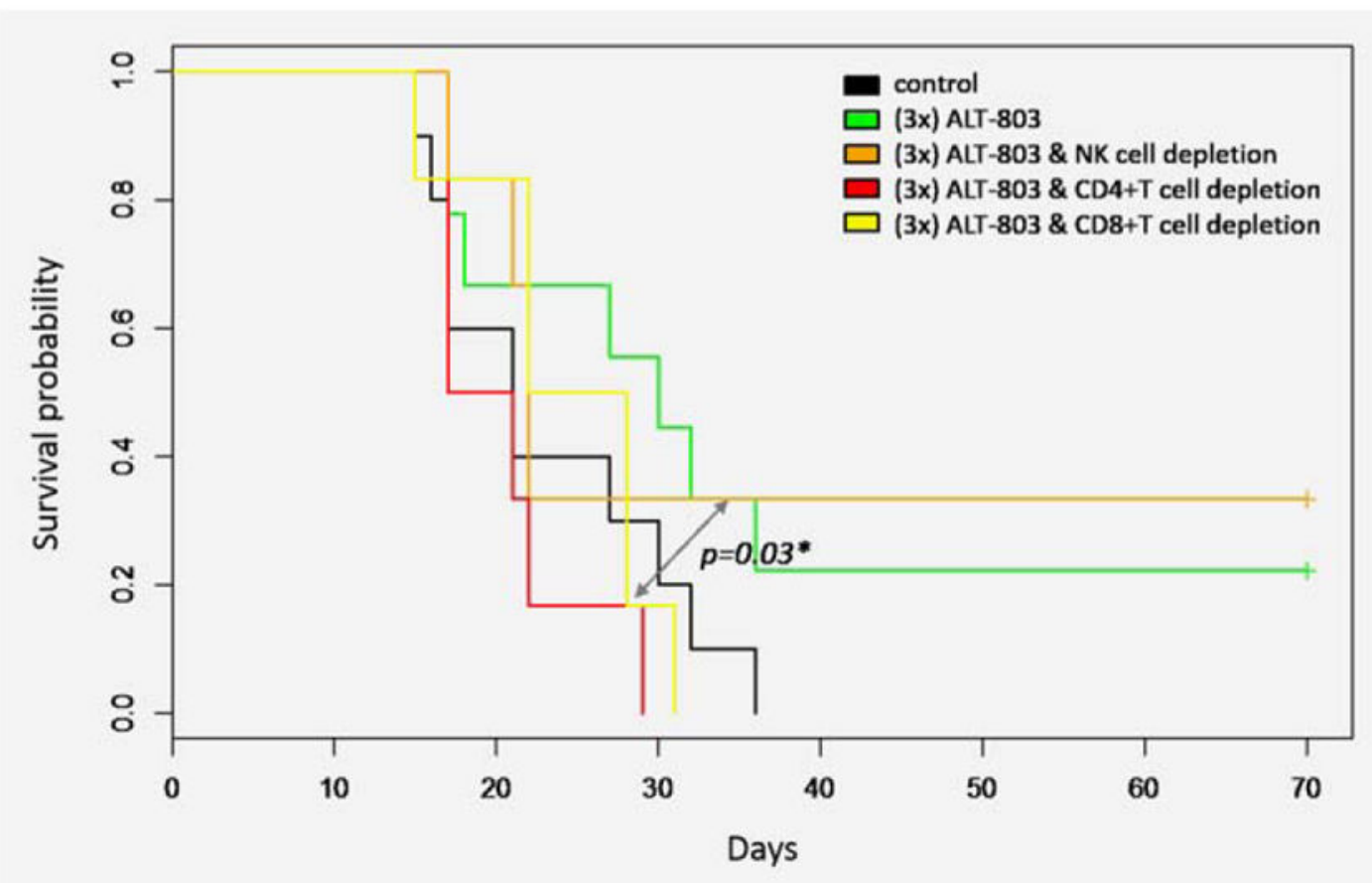

Figure 4.

The antitumor effect of ALT-803 against glioblastoma is mediated by CD4+ and CD8+ T cells. CD4 and CD8 depletion but not NK depletion abrogates the survival benefit of ALT-803. Mice were depleted of different immune cell populations before the initiation of ALT-803 treatment and the immune cell depletion was maintained by giving additional depletion antibody injections after the end of the ALT-803 treatment. [Color figure can be viewed in the online issue, which is available at wileyonlinelibrary.com.] 

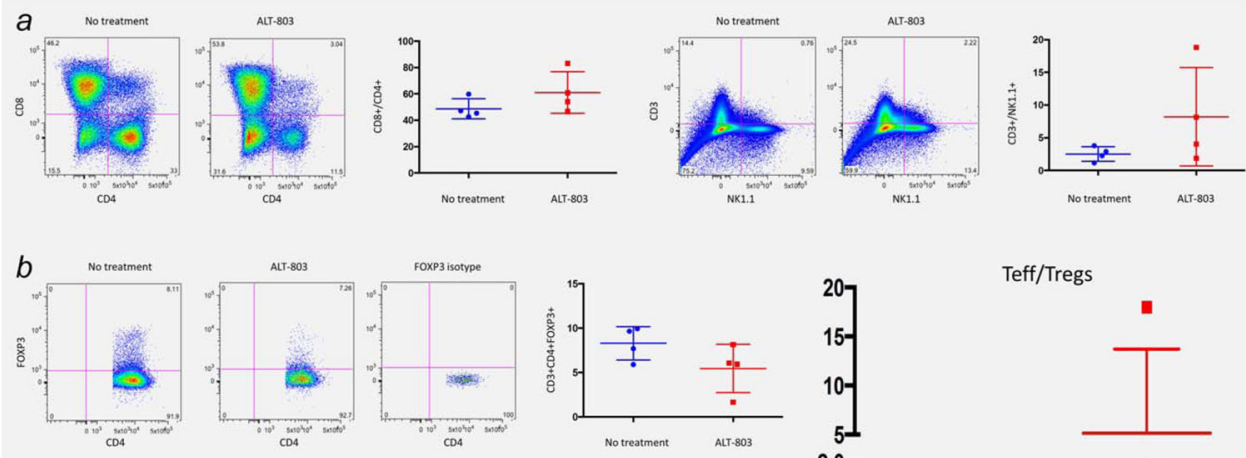

Teff/Tregs
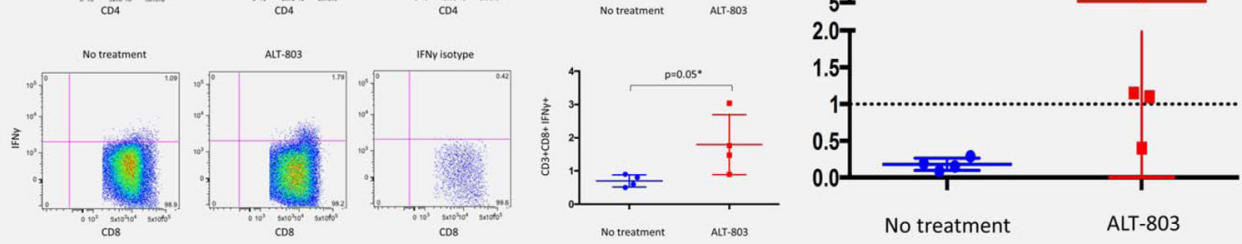

Figure 5.

ALT-803 treated mice are exhibiting increased infiltration of immune cells with an activated phenotype. (A) ALT-803 increases the infiltration of brain tumor by tumor infiltrating lymphocytes and natural killer cells, while it is preferentially increasing the percentage of CD8+ T cells in the immune infiltrate compared to CD4+ T cells. ALT-803 is increasing the ratio of $\mathrm{CD} 3+/ \mathrm{NK}$ cells ( 8 vs $2, p=0.1$ ) in the tumor microenviroment and is increasing the percentage of tumor infiltrating CD8+ T cells $(50 \%$ vs $62 \%, p=0.56)$. (B) ALT-803 increases the infiltration of $\mathrm{CD} 8+/ \mathrm{IFN} \gamma+$ effector $\mathrm{T}$ cells $\left(\mathrm{T}_{\text {eff }}\right)$ significantly ( $1.8 \%$ vs $0.8 \%$, $p=0.05)$ and decreases the percentage of CD4+/FoxP3+ regulatory $\mathrm{T}$ cells $\left(\mathrm{T}_{\text {reg }}\right)(7.8 \mathrm{vs}$ $5.3, p=0.4)$. As a result, the ratio of $\mathrm{T}_{\text {eff }} / \mathrm{T}_{\text {reg }}$ is higher in the ALT-803 treated compared to the control mice ( 6 vs $0.1, p=0.2$ ). [Color figure can be viewed in the online issue, which is available at wileyonlinelibrary.com.] 